\title{
Nonseparable two-dimensional fractional Fourier transform
}

\author{
Aysegul Sahin, M. Alper Kutay, and Haldun M. Ozaktas
}

\begin{abstract}
Previous generalizations of the fractional Fourier transform to two dimensions assumed separable kernels. We present a nonseparable definition for the two-dimensional fractional Fourier transform that includes the separable definition as a special case. Its digital and optical implementations are presented. The usefulness of the nonseparable transform is justified with an image-restoration example. (C) 1998 Optical Society of America
\end{abstract}

OCIS codes: $100.0100,070.2590,100.3020$.

\section{Introduction}

The fractional Fourier transform of the order $a_{1}$ is defined in a manner such that the common Fourier transform is a special case with the order $a_{1}=1$. The one-dimensional (1-D) fractional Fourier transform of the order $a_{1}$ can be defined for $0<\left|a_{1}\right|<2$ as

$$
\begin{aligned}
\mathscr{F}^{a_{1}}[f(x)](x)= & \int_{-\infty}^{\infty} B_{a_{1}}\left(x, x^{\prime}\right) f\left(x^{\prime}\right) \mathrm{d} x^{\prime} \\
B_{a_{1}}\left(x, x^{\prime}\right)= & A_{\phi_{1}} \exp \left[i \pi \left(x^{2} \cot \phi_{1}-2 x x^{\prime} \csc \phi_{1}\right.\right. \\
& \left.\left.+x^{\prime 2} \cot \phi_{1}\right)\right]
\end{aligned}
$$

where $A_{\phi_{1}}=\exp \left[-i\left(\pi \hat{\phi}_{1} / 4-\hat{\phi}_{1} / 2\right)\right] /\left|\sin \phi_{1}\right|^{1 / 2}, \phi_{1}=$ $a_{1} \pi / 2$, and $\hat{\phi}_{1}=\operatorname{sgn}\left(\phi_{1}\right)$. The kernel is defined separately for $a_{1}=0$ and $a_{1}= \pm 2$ as $B_{0}\left(x, x^{\prime}\right)=\delta(x-$ $\left.x^{\prime}\right)$ and $B_{ \pm 2}\left(x, x^{\prime}\right)=\delta\left(x+x^{\prime}\right)$. The definition can easily be extended outside the interval $[-2,2]$ if we note that $\mathscr{F}^{4 j+a_{1}}(x)=\mathscr{F}_{\mathscr{f}} a_{1}(x) .{ }^{1}$

Some essential properties of the fractional Fourier transform are (i) it is linear, (ii) the first-order transform $\left(a_{1}=1\right)$ corresponds to the common Fourier transform, and (iii) it is additive in index, $\mathscr{F}^{a_{1}} \mathscr{F}^{b_{1}} \hat{q}=$ $\mathscr{F}^{a_{1}+b_{1}} \hat{q}$. The kernel of the inverse transform is given by $B_{a_{1}}{ }^{-1}\left(x, x^{\prime}\right)=B_{-a_{1}}\left(x, x^{\prime}\right)=B_{a_{1}}{ }^{*}\left(x, x^{\prime}\right)$. Other properties can be found in Refs. 1-8.

The fractional Fourier transform can be realized optically like the ordinary Fourier transformation. ${ }^{3,4,9-14}$ Thus it has many applications in optical signal processing. ${ }^{3-6,9-11,13-22}$

The authors are with the Department of Electrical Engineering, Bilkent University, TR-06533 Bilkent, Ankara, Turkey.

Received 4 December 1997; revised manuscript received 12 May 1998.

0003-6935/98/235444-10\$15.00/0

(C) 1998 Optical Society of America
The fractional Fourier transform definition can easily be extended to two dimensions if one assumes a separable kernel. ${ }^{4,12-14,23}$ These definitions have separable kernels and possess properties similar to the 1-D transform. The separable two-dimensional (2-D) fractional Fourier transform of the orders $a_{1}$ for the $x$ axis and $a_{2}$ for the $y$ axis for $0<\left|a_{1}\right|<2$ and $0<$ $\left|a_{2}\right|<2$, respectively, is defined as

$$
\begin{aligned}
& \mathscr{F}^{a_{1}, a_{2}}[f(x, y)](x, y) \\
& \quad=\int_{-\infty}^{\infty} \int_{-\infty}^{\infty} B_{a_{1}, a_{2}}\left(x, y ; x^{\prime}, y^{\prime}\right) f\left(x^{\prime}, y^{\prime}\right) \mathrm{d} x^{\prime} \mathrm{d} y^{\prime},
\end{aligned}
$$

where

$$
B_{a_{1}, a_{2}}\left(x, y ; x^{\prime}, y^{\prime}\right)=B_{a_{1}}\left(x, x^{\prime}\right) B_{a_{2}}\left(y, y^{\prime}\right) .
$$

Both $x$ and $y$ are interpreted as dimensionless variables.

The properties and optical implementations of the 2-D fractional Fourier transform are given in Refs. 12, 14, 24, and 25. The separable 2-D fractional Fourier transform is nothing but a repetition of the transform in the $x$ and the $y$ directions independently and is not the most general definition possible in two dimensions. In this paper we propose a nonseparable definition for the 2-D fractional Fourier transform that is more general than the separable one. We first explain our motivation in looking for a new, nonseparable transform. After giving the new definition, we derive its properties. Both the digital and optical implementations of the nonseparable definition are presented. Finally, we use an image-restoration example to justify the usefulness of the nonseparable definition by showing that better performance is obtained compared with the separable definition. 


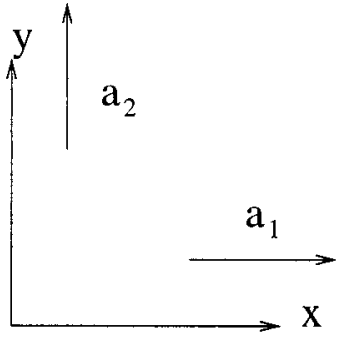

(a)

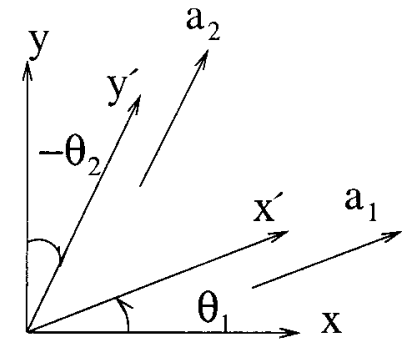

(b)
Fig. 1. Transform orders and directions for (a) the separable transform and (b) the nonseparable transform.

\section{Motivation}

Many properties of the Fourier transform generalize trivially to two dimensions, but new properties exist in two dimensions, such as the following: If $f(x, y)$ has a 2-D Fourier transform $F(x, y)$, then $f(a x+b y$, $c x+d y$ ) has a 2-D Fourier transform given by

$$
G(x, y)=\frac{1}{\Delta} F\left(\frac{d x-c y}{\Delta}, \frac{-b x+a y}{\Delta}\right),
$$

where $\Delta=a d-b c .^{26}$ Because the Fourier transform is a special case of the fractional Fourier transform, we look for a similar property for the 2-D fractional Fourier transform; however, the 2-D fractional Fourier transform does not have a similar property. If $F_{a_{1}, a_{2}}(x, y)$ is the 2-D fractional Fourier transform of $f(x, y)$ with the orders $a_{1}$ and $a_{2}$, then $G_{a_{1}, a_{2}}(x, y)$, which is the 2-D fractional Fourier transform of $f(a x+b y, c x+d y)$, cannot be represented in terms of a scaled version of $F_{a_{1}, a_{2}}(x, y)$ with a relation similar to that of Eq. (5). This is one of our motivations for searching for a new nonseparable definition.

The separable definition has two parameters, $a_{1}$ and $a_{2}$. The function is fractionally Fourier transformed along the $x$ and the $y$ axes with the orders $a_{1}$

tion for us to look for a new definition for the 2-D fractional Fourier transform.

\section{Nonseparable Fractional Fourier Transform}

Here we present our new, nonseparable definition for the 2-D fractional Fourier transform. We define the nonseparable fractional Fourier transform in such a manner that it corresponds to the fractional Fourier transformation along the arbitrary $x^{\prime}$ and $y^{\prime}$ directions with the orders $a_{1}$ and $a_{2}$, respectively. It is equivalent to the rotation of the $x$ and the $y$ axes followed by the separable definition. First, the $x$ axis is rotated by an angle $\theta_{1}$, and the $y$ axis is rotated by an angle $\theta_{2}$. Thus the $x$ axis is mapped to $x^{\prime}$, which makes the angle $\theta_{1}$ with the $x$ axis, and the $y$ axis is mapped to $y^{\prime}$, which makes the angle $\theta_{2}$ with the $y$ axis. This is equivalent to mapping $f(x, y)$ to $f\left[\left(\cos \theta_{1} x+\sin \theta_{1} y\right) / \cos \left(\theta_{1}-\theta_{2}\right),\left(-\sin \theta_{2} x+\right.\right.$ $\left.\left.\cos \theta_{2} y\right) / \cos \left(\theta_{1}-\theta_{2}\right)\right]$. Then the 2-D separable fractional Fourier transform operator with the orders $a_{1}$ and $a_{2}$ is applied to $f\left[\left(\cos \theta_{1} x+\sin \theta_{1} y\right) / \cos \left(\theta_{1}-\theta_{2}\right)\right.$, $\left.\left(-\sin \theta_{2} x+\cos \theta_{2} y\right) / \cos \left(\theta_{1}-\theta_{2}\right)\right]$. The resulting transformation is the new, nonseparable 2-D fractional Fourier transform.

The new definition has four parameters: $a_{1}, a_{2}$, $\theta_{1}$, and $\theta_{2}$. The parameter $\theta_{1}$ is the angle between the $x$ axis and the $x^{\prime}$ axis, $a_{1}$ is the order specified along the $x^{\prime}$ direction, $\theta_{2}$ is the angle between the $y$ axis and the $y^{\prime}$ axis, and $a_{2}$ is the order along the $y^{\prime}$ direction. The nonseparable fractional Fourier transform can be written mathematically as

$$
\mathscr{F}_{\theta_{1}, \theta_{2}}^{a_{1}, a_{2}}[f(\mathbf{r})](\mathbf{r})=\int_{-\infty}^{\infty} \int_{-\infty}^{\infty} B_{a_{1}, a_{2}, \theta_{1}, \theta_{2}}\left(\mathbf{r}, \mathbf{r}^{\prime \prime}\right) f\left(\mathbf{r}^{\prime \prime}\right) \mathrm{d} \mathbf{r}^{\prime \prime},
$$

where

$$
\begin{aligned}
B_{a_{1}, a_{2}, \theta_{1}, \theta_{2}}\left(\mathbf{r}, \mathbf{r}^{\prime \prime}\right) & \\
& =A_{\phi_{1}, \phi_{2}} \exp \left[i \pi\left(\mathbf{r}^{T} \mathbf{A} \mathbf{r}+2 \mathbf{r}^{T} \mathbf{B} \mathbf{r}^{\prime \prime}+\mathbf{r}^{\prime \prime T} \mathbf{C r}^{\prime \prime}\right)\right], \\
A_{\phi_{1}, \phi_{2}} & =A_{\phi_{1}} A_{\phi_{2}}, \quad \mathbf{r}=[x y]^{T}, \quad \mathbf{r}^{\prime \prime}=\left[x^{\prime \prime} y^{\prime \prime}\right]^{T},
\end{aligned}
$$

$$
\left.\begin{array}{c}
-\frac{\sin \theta_{1} \cos \theta_{2}}{\cos ^{2}\left(\theta_{1}-\theta_{2}\right)} \cot \phi_{1}+\frac{\sin \theta_{2} \cos \theta_{1}}{\cos ^{2}\left(\theta_{1}-\theta_{2}\right)} \cot \phi_{2} \\
\frac{\cos ^{2} \theta_{1}}{\cos ^{2}\left(\theta_{1}-\theta_{2}\right)} \cot \phi_{2}+\frac{\sin ^{2} \theta_{1}}{\cos ^{2}\left(\theta_{1}-\theta_{2}\right)} \cot \phi_{1}
\end{array}\right] .
$$

and $a_{2}$, respectively, as shown in Fig. 1(a). More generally, we wish to specify both the directions $x^{\prime}$ and $y^{\prime}$ and the orders $a_{1}$ and $a_{2}$ of the 2-D transform, as can be seen in Fig. 1(b). This is another motiva-
Here it is important to note that $x^{\prime}$ and $y^{\prime}$ determine the directions along which we specify the orders, whereas $x^{\prime \prime}$ and $y^{\prime \prime}$ are merely dummy variables. We denote this nonseparable fractional Fourier operator 
by $\mathscr{F}_{\theta_{1}, \theta_{2}} a_{1}, a_{2}$, whereas the separable transform is denoted by $\mathscr{F}^{a_{1}, a_{2}}$. The term $\mathscr{F}_{\theta_{1}, \theta_{2}}^{a_{1}, a_{2}}$ reduces to $\mathscr{F}^{a_{1}, a_{2}}$ if one chooses $\theta_{1}=\theta_{2}=0$.

This definition with four parameters is specified by its nonseparable kernel. We constructed the definition in such a way that it corresponds to the fractional Fourier transformation along arbitrary $x^{\prime}$ and $y^{\prime}$ directions. The following theorem states that, when a linear distortion is applied to the function, its nonseparable fractional Fourier transform can be represented in terms of the linearly distorted form of the transform of the original function.

Theorem 1. The nonseparable fractional Fourier transform of $f(a x+b y, c x+d y)$ with the orders $a_{1}$ and $a_{2}$ can be represented in terms of the nonseparable fractional Fourier transform of $f(x, y)$ as
Fourier transform despite the fact that considerable algebraic manipulations are involved.

We mentioned in Section 2 that the nonseparable definition is a generalization of the separable definition. The reader may wonder whether even more general definitions that employ more than four parameters are also possible. For instance, we might propose a six-parameter transform defined as the separable fractional Fourier transform of $f(a x+b y$, $c x+d y)$ with the orders $a_{1}$ and $a_{2}$. We now show that such a definition is redundant because the separable fractional Fourier transform of $f(a x+b y, c x+$ $d y$ ) for any parameters $a_{1}, a_{2}, a, b, c$, and $d$ can be represented as a scaled version of the four-parameter nonseparable transform.

$$
\begin{aligned}
\mathscr{F}_{\theta_{1}, \theta_{2}}^{a_{1}, a_{2}}[f(a x+b y, c x+d y)](x, y) & =k \mathscr{F}_{\theta_{1}^{\prime}, \theta_{2}^{\prime}}^{a a_{2}^{\prime}}[f(x, y)]\left(a^{\prime} x+b^{\prime} y, c^{\prime} x+d^{\prime} y\right), \\
k & =\exp \left(C_{\phi_{1}} x^{2}+C_{\phi_{2}} y^{2}+C_{\phi_{1}^{\prime}, \phi_{2}} x y\right), \\
\phi_{1}^{\prime} & =\phi_{1} \cot ^{-1}\left[\frac{D_{\theta_{1} \theta_{2}}}{\left(a \cos \theta_{1}+b \sin \theta_{2}\right)^{2}-\left(c \cos \theta_{1}+d \sin \theta_{2}\right)^{2}}\right], \\
\phi_{2}^{\prime} & =\phi_{2} \cot ^{-1}\left[\frac{D_{\theta_{1} \theta_{2}}}{\left(d \cos \theta_{2}+c \sin \theta_{1}\right)^{2}-\left(b \cos \theta_{2}+c \sin \theta_{1}\right)^{2}}\right], \\
\theta_{1}^{\prime} & =\cos ^{-1}\left\{\frac{\left.\left(a \cos \theta_{1}+b \sin \theta_{2}\right)^{2}\left[\left(d \cos \theta_{2}+c \sin \theta_{1}\right)^{2}-\left(b \cos \theta_{2}+a \sin \theta_{1}\right)^{2}\right]\right\}^{1 / 2}}{D_{\theta_{1} \theta_{2}}},\right. \\
\theta_{2}^{\prime} & =\cos ^{-1}\left\{\frac{\left(d \cos \theta_{2}+c \sin \theta_{1}\right)^{2}\left[\left(a \cos \theta_{1}+b \sin \theta_{2}\right)^{2}-\left(c \cos \theta_{1}+d \sin \theta_{2}\right)^{2}\right]}{D_{\theta_{1} \theta_{2}}}\right\}^{1 / 2}, \\
a^{\prime} & =\frac{\csc _{\phi_{1}}\left[\left(d \cos \theta_{2}+c \sin \theta_{1}\right) \cos \theta_{1}+\left(b \cos \theta_{2}+a \sin \theta_{2}\right) \sin \theta_{2}\right]}{\csc \phi_{1}^{\prime} \cos \left(\theta_{1}-\theta_{2}\right)}, \\
b^{\prime} & =\frac{\csc \phi_{2}\left[\left(c \cos \theta_{1}+d \sin \theta_{2}\right) \cos \theta_{1}+\left(a \cos \theta_{1}+b \sin \theta_{2}\right) \sin \theta_{2}\right]}{\csc \phi_{1}^{\prime} \cos \left(\theta_{1}-\theta_{2}\right)} \\
c^{\prime} & =\frac{\csc \phi_{1}\left[\left(d \cos \theta_{2}+c \sin \theta_{1}\right) \sin \theta_{1}-\left(b \cos \theta_{2}+a \sin \theta_{1}\right) \cos \theta_{2}\right]}{\csc \phi_{2}^{\prime} \cos \left(\theta_{1}-\theta_{2}\right)} \\
d^{\prime} & =\frac{\csc \phi_{2}\left[\left(a \cos \theta_{1}+b \sin \theta_{2}\right) \cos \theta_{2}-\left(c \cos \theta_{1}+d \sin \theta_{2}\right) \sin \theta_{1}\right]}{\csc \phi_{2}^{\prime} \cos \left(\theta_{1}-\theta_{2}\right)},
\end{aligned}
$$

where we employ the intermediate variables

$$
\begin{aligned}
D_{\theta_{1} \theta_{2}} & =\left[\left(a \cos \theta_{1}+b \sin \theta_{2}\right)^{2}\left(d \cos \theta_{2}+c \sin \theta_{1}\right)^{2}-\left(b \cos \theta_{2}+a \sin \theta_{1}\right)^{2}\left(c \cos \theta_{1}+d \sin \theta_{2}\right)\right], \\
C_{\phi_{1}} & =\frac{\cot \phi_{1}\left\{\left[\left(a \cos \theta_{1}+b \sin \theta_{2}\right)^{2}-\left(c \cos \theta_{1}+d \sin \theta_{2}\right)^{2}\right]-D_{\theta_{1} \theta_{2}}\right\}}{\left\{\left[\left(a \cos \theta_{1}+b \sin \theta_{2}\right)^{2}-\left(c \cos \theta_{1}+d \sin \theta_{2}\right)^{2}\right]-\cot ^{2} \phi_{1} D_{\theta_{1} \theta_{2}}\right\}}, \\
C_{\phi_{2}} & =\frac{\cot \phi_{2}\left\{\left[\left(d \cos \theta_{2}+c \sin \theta_{1}\right)^{2}-\left(b \cos \theta_{2}+c \sin \theta_{1}\right)^{2}\right]-D_{\theta_{1} \theta_{2}}\right\}}{\left\{\left[\left(d \cos \theta_{2}+c \sin \theta_{1}\right)^{2}-\left(b \cos \theta_{2}+a \sin \theta_{1}\right)^{2}\right]-\cot ^{2} \phi_{2} D_{\theta_{1} \theta_{2}}\right\}}, \\
C_{\phi_{1}^{\prime}, \phi_{2}^{\prime}} & =a^{\prime} b^{\prime} \cot \phi_{1}^{\prime}+c^{\prime} d^{\prime} \cot \phi_{2}^{\prime} .
\end{aligned}
$$

Proof: The proof of this property follows directly from the definition of the nonseparable fractional
Theorem 2: The fractional Fourier transform of $f(a x+b y, c x+d y)$ with the orders $a_{1}$ and $a_{2}$, accord- 
ing to the separable definition, can be represented as a scaled version of the nonseparable fractional Fourier transform of $f(x, y)$ :
Likewise, a definition for the 2-D fractional Fourier transform with more than four parameters will be redundant.

$$
\begin{array}{cc}
\mathscr{F}^{a_{1}, a_{2}}[f(a x+b y, c x+d y)](x, y)=k \mathscr{F}_{\theta_{1}, \theta_{2}^{\prime}}^{a a_{2}^{\prime}}[f(x, y)]\left(a^{\prime} x+b^{\prime} y, c^{\prime} x+d^{\prime} y\right), \\
\Delta=(a d-b c), \quad k=\frac{1}{\Delta} \exp \left[C_{\phi_{1}} x^{2}+C_{\phi_{2}} y^{2}+C_{\phi_{1}^{\prime}, \phi_{2}^{\prime}} x y\right], \\
\phi_{1}^{\prime}=\phi_{1} \cot ^{-1}\left[\frac{\left(a^{2} d^{2}-b^{2} c^{2}\right)}{\Delta^{2}\left(a^{2}-c^{2}\right)}\right], & \phi_{2}^{\prime}=\phi_{2} \cot ^{-1}\left[\frac{\left(a^{2} d^{2}-b^{2} c^{2}\right)}{\Delta^{2}\left(d^{2}-b^{2}\right)}\right], \\
\theta_{1}^{\prime}=\cos ^{-1}\left[\frac{a^{2}\left(d^{2}-b^{2}\right)}{\left(a^{2} d^{2}-b^{2} c^{2}\right)}\right]^{1 / 2}, & \theta_{2}^{\prime}=\cos ^{-1}\left[\frac{d^{2}\left(a^{2}-c^{2}\right)}{\left(a^{2} d^{2}-b^{2} c^{2}\right)}\right]^{1 / 2}, \\
a^{\prime}=\frac{\csc \phi_{1}\left(d \cos \theta_{1}+b \sin \theta_{2}\right)}{\Delta \csc \phi_{1}^{\prime} \cos \left(\theta_{1}-\theta_{2}\right)}, & b^{\prime}=\frac{\csc \phi_{2}\left(c \cos \theta_{1}+a \sin \theta_{2}\right)}{\Delta \csc \phi_{2}^{\prime} \cos \left(\theta_{1}-\theta_{2}\right)}, \\
c^{\prime}=\frac{\csc \phi_{1}\left(d \sin \theta_{1}-b \cos \theta_{2}\right)}{\Delta \csc \phi_{2}^{\prime} \cos \left(\theta_{1}-\theta_{2}\right)}, & d^{\prime}=\frac{\csc \phi_{2}\left(a \cos \theta_{2}-c \sin \theta_{1}\right)}{\Delta \csc \phi_{2}^{\prime} \cos \left(\theta_{1}-\theta_{2}\right)},
\end{array}
$$

where the intermediate variables are

$$
\begin{aligned}
k & =\frac{1}{\Delta} \exp \left(C_{\phi_{1}} x^{2}+C_{\phi_{2}} y^{2}+C_{\phi_{1}^{\prime}, \phi_{2}^{\prime}} x y\right), \\
\Delta & =(a d-b c), \\
C_{\phi_{1}} & =\frac{\cot \phi_{1}\left[\Delta^{4}\left(a^{2}-c^{2}\right)-\left(a^{2} d^{2}-b^{2} c^{2}\right)^{2}\right]}{\left[\Delta^{4}\left(a^{2}-c^{2}\right)-\cot ^{2} \phi_{1}\left(a^{2} d^{2}-b^{2} c^{2}\right)^{2}\right]}, \\
C_{\phi_{2}} & =\frac{\cot \phi_{2}\left[\Delta^{4}\left(d^{2}-b^{2}\right)-\left(a^{2} d^{2}-b^{2} c^{2}\right)^{2}\right]}{\left[\Delta^{4}\left(d^{2}-b^{2}\right)-\cot ^{2} \phi_{2}\left(a^{2} d^{2}-b^{2} c^{2}\right)^{2}\right]}, \\
C_{\phi_{1}^{\prime}, \phi_{2}^{\prime}} & =a^{\prime} b^{\prime} \cot \phi_{1}^{\prime}+c^{\prime} d^{\prime} \cot \phi_{2}^{\prime} .
\end{aligned}
$$

Proof: This theorem can easily be proved by use of the definitions of the separable and the nonseparable 2-D fractional Fourier transforms through straightforward yet lengthy algebraic manipulations.

Theorem 2 states that the separable fractional Fourier transform of any function $f(a x+b y, c x+$ $d y$ ) can be represented as a linearly distorted version of the nonseparable fractional Fourier transform of the original function. This result indicates that the six-parameter definition is redundant. An analogy with the common Fourier transform might be useful. We know that, when a function is scaled, its Fourier transform can be represented as a scaled version of the Fourier transform of the original function. Thus it is redundant to define a transform called the "scaled Fourier transform."

\section{Properties of the Nonseparable Fractional Fourier Transform}

Theorem 3: The kernel of the inverse transform is

$$
\begin{aligned}
B_{a_{1}, a_{2}, \theta_{1}, \theta_{2}}^{-1}\left(\mathbf{r}, \mathbf{r}^{\prime \prime}\right)= & A_{-\phi_{1},-\phi_{2}} \exp \left[-i \pi\left(\mathbf{r}^{T} \mathbf{C r}+2 \mathbf{r}^{T} \mathbf{B}^{T} \mathbf{r}^{\prime \prime}\right.\right. \\
& \left.\left.+\mathbf{r}^{\prime \prime T} \mathbf{A} \mathbf{r}^{\prime \prime}\right)\right],
\end{aligned}
$$

where $\mathbf{A}, \mathbf{B}$, and $\mathbf{C}$ are given in Eqs. (8)-(10). Note that the kernel of the inverse transform $B_{a_{1}, a_{2}, \theta_{1}, \theta_{2}}^{-1}(\mathbf{r}$, $\left.\mathbf{r}^{\prime \prime}\right)$ is not equivalent to $B_{-a_{1},-a_{2},-\theta_{1},-\theta_{2}}\left(\mathbf{r}, \mathbf{r}^{\prime \prime}\right)$ or $B_{-a_{1},-a_{2}, \theta_{1}, \theta_{2}}(\mathbf{r}, \mathbf{r})$.

Proof: We know that the fractional Fourier transform according to the new definition can be decomposed into an affine transform followed by the separable definition. Thus

$$
\begin{aligned}
\mathscr{F}_{\theta_{1}, \theta_{2}}^{a_{1}, a_{2}}[f(x, y)]= & \mathscr{F}^{a_{1}, a_{2}}\left\{f \left[\left(\cos \theta_{1} x+\sin \theta_{1} y\right) / \cos \left(\theta_{1}-\theta_{2}\right),\right.\right. \\
& \left.\left.\left(-\sin \theta_{2} x+\cos \theta_{2} y\right) / \cos \left(\theta_{1}-\theta_{2}\right)\right]\right\} .
\end{aligned}
$$

By using the inverse kernel given in Eq. (3) and applying the inverse of the linear coordinate distortion, we find that the result follows easily.

Theorem 4: The nonseparable definition is unitary:

$$
B_{a_{1}, a_{2}, \theta_{1}, \theta_{2}}^{*}\left(x, y ; x^{\prime \prime}, y^{\prime \prime}\right)=B_{a_{1}, a_{2}, \theta_{1}, \theta_{2}}^{-1}\left(x^{\prime \prime}, y^{\prime \prime} ; x, y\right) .
$$

Proof: By using the kernel of the nonseparable transform in Eq. (6) and its inverse in Eq. (12), we find that the proof follows.

Theorem 5: Let $W_{f}\left(x, y ; \mu_{x}, \mu_{y}\right)$ be the Wigner distribution of $f(x, y)$. If $g(x, y)$ is the nonseparable fractional Fourier transform of $f(x, y)$ with the pa- 
rameters $a_{1}, a_{2}, \theta_{1}$, and $\theta_{2}$, then the Wigner distribution of $g(x, y)$ is related to that of $f(x, y)$ through the following:

$$
\begin{aligned}
W_{g}(\mathbf{r}, \mu) & =W_{f}\left(\mathbf{A r}+\mathbf{B} \mu, \mathbf{C r}+\mathbf{D}_{\mu}\right), \\
\mathbf{r} & =\left[\begin{array}{ll}
x & y
\end{array}\right]^{T}, \quad \mu=\left[\begin{array}{ll}
\mu_{x} & \mu_{y}
\end{array}\right]^{T},
\end{aligned}
$$

where

$$
\begin{aligned}
& \mathbf{A}=\frac{1}{\cos \left(\theta_{1}-\theta_{2}\right)}\left[\begin{array}{cc}
\cos \phi_{1} \cos \theta_{1} & \cos \phi_{2} \sin \theta_{1} \\
-\cos \phi_{1} \sin \theta_{2} & \cos \phi_{2} \cos \theta_{2}
\end{array}\right], \\
& \mathbf{B}=\frac{1}{\cos \left(\theta_{1}-\theta_{2}\right)}\left[\begin{array}{cc}
-\sin \phi_{1} \cos \theta_{1} & -\sin \phi_{2} \sin \theta_{1} \\
\sin \phi_{1} \sin \theta_{2} & -\sin \phi_{2} \cos \theta_{2}
\end{array}\right], \\
& \mathbf{C}=\frac{1}{\cos \left(\theta_{1}-\theta_{2}\right)}\left[\begin{array}{cc}
\sin \phi_{1} \cos \theta_{2} & \sin \phi_{2} \sin \theta_{2} \\
-\sin \phi_{1} \sin \theta_{1} & \sin \phi_{2} \cos \theta_{1}
\end{array}\right], \\
& \mathbf{D}=\frac{1}{\cos \left(\theta_{1}-\theta_{2}\right)}\left[\begin{array}{cc}
\cos \phi_{1} \cos \theta_{2} & \cos \phi_{2} \sin \theta_{2} \\
-\cos \phi_{1} \sin \theta_{1} & \cos \phi_{2} \cos \theta_{1}
\end{array}\right]
\end{aligned}
$$

Proof: This is again a direct consequence of the definition of the nonseparable transform and the definition of the Wigner distribution. The Wigner distribution is defined as

$$
W(\mathbf{r}, \mu)=\int_{-\infty}^{\infty} f\left(\mathbf{r}+\frac{\mathbf{r}^{\prime}}{2}\right) f^{*}\left(\mathbf{r}-\frac{\mathbf{r}^{\prime}}{2}\right) \exp \left(i 2 \pi \mu^{T} \mathbf{r}^{\prime}\right) \mathrm{d} \mathbf{r}^{\prime}
$$

where

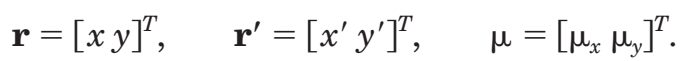

The relation between the Wigner distribution of a 2-D function and the Wigner distribution of the affinetransformed version of that 2-D function is given in Ref. 27. We also know the effect of the separable 2-D fractional Fourier transform on the Wigner distribution. ${ }^{24}$ The above result follows easily from these facts when they are applied succesively. Results of a similar nature for various kinds of transforms are discussed in Ref. 28.

It should be noted that the nonseparable transform discussed in this paper is not a fractional operator in the strict sense. For $\theta_{1}=\theta_{2}=0$, it does correspond to the identity transform and the Fourier transform when $a_{1}=a_{2}=0$ and $a_{1}=a_{2}=1$, respectively. However, the operator $\mathscr{F}_{\theta_{1}, \theta_{2}}^{a_{1}, a_{2}}$ is not the fractional power of $\mathscr{F}_{\theta_{1}, \theta_{2}}^{1,1}$. Nevertheless, because the transform is motivated by the desire to fractional Fouriertransform an image along directions other than the orthogonal $x$ and $y$ axes and because its applications are natural extensions of those of the separable fractional Fourier transform, we find it appropriate to

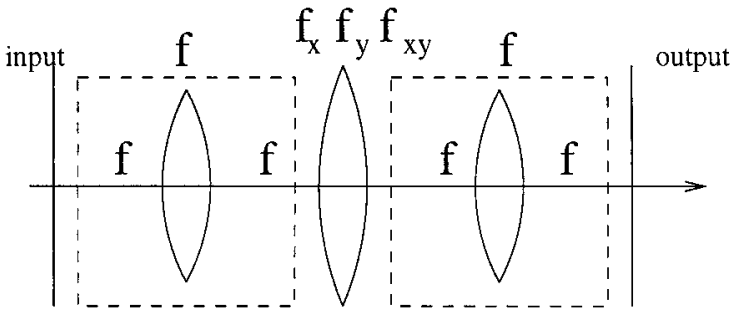

Fourier

Block

Inverse Fourier

Block

Fig. 2. Optical setup for simulating anamorphic sections of free space.

refer to it as the nonseparable fractional Fourier transform.

\section{Digital Implementation of the Nonseparable Transform}

Because of the oscillatory nature of the fractional Fourier transform, its digital implementation is time consuming with simple integration techniques. However, in Ref. 29 a fast algorithm for the fractional Fourier transform is presented. Although direct computation would require $O\left(N^{2}\right)$ multiplications, this fast algorithm computes the transform in $O(N \log N)$ time.

To use the nonseparable definition for practical purposes, we need a fast digital implementation. By definition, it is composed of a linear distribution that is followed by the separable definition. In image processing several algorithms exist for linear distortions. ${ }^{30,31}$ To implement the nonseparable fractional Fourier transform of $f(x, y)$ with the parameters $a_{1}, a_{2}, \theta_{1}$, and $\theta_{2}$, we first compute $f\left[\left(\cos \theta_{1} x+\right.\right.$ $\left.\left.\sin \theta_{1} y\right) / \cos \left(\theta_{1}-\theta_{2}\right),\left(-\sin \theta_{2} x+\cos \theta_{2} y\right) / \cos \left(\theta_{1}-\theta_{2}\right)\right]$. This is achieved by use of the bilinear interpolation method. Then the fast algorithm given in Ref. 29 is applied. The resulting transformation is the nonseparable fractional Fourier transform. The computation time is again of the order of $O(N \log N)$, where $N$ is the number of pixels in the 2-D function (image).

\section{Optical Implementation of the Nonseparable Transform}

To implement the nonseparable fractional Fourier transform optically, it is necessary to employ anamorphic or cylindrical lenses and anamorphic sections of free space. When a distribution of light passes through an anamorphic lens, it is multiplied by the function

$$
\exp \left[-i \pi\left(\frac{x^{2}}{\lambda f_{x}}+\frac{y^{2}}{\lambda f_{y}}+\frac{x y}{\lambda f_{x y}}\right)\right],
$$

where $f_{x}, f_{y}$, and $f_{x y}$ are the parameters of the lens. Such a lens can be simulated by two cylindrical lenses that make appropriate angles with the $x$ and the $y$ axes. When a distribution of light propagates 
through an anamorphic free space, it is convolved by the function

$$
\exp \left[i \pi\left(\frac{x^{2}}{\lambda d_{x}}+\frac{y^{2}}{\lambda d_{y}}+\frac{x y}{\lambda d_{x y}}\right)\right],
$$

where $d_{x}, d_{y}$, and $d_{x y}$ are the parameters of the anamorphic free space. Such anamorphic sections of free space can be simulated by use of anamorphic lenses, as shown in Fig. 2. This is based on the fact that convolution in the space domain corresponds to multiplication in the Fourier domain. The parameters $d_{x}, d_{y}$, and $d_{x y}$ of the anamorphic sections of free space that we are simulating are related to the parameters of the anamorphic lens $f_{x}, f_{y}$, and $f_{x y}$, according to

$$
\begin{gathered}
d_{x}=\frac{s^{4}\left(f_{x y}^{2}-f_{x} f_{y}\right)}{\lambda^{2} f_{x} f_{x y}^{2}}, \quad d_{y}=\frac{s^{4}\left(f_{x y}^{2}-f_{x} f_{y}\right)}{\lambda^{2} f_{y} f_{x y}^{2}}, \\
d_{x y}=\frac{s^{4}\left(f_{x} f_{y}-f_{x y}^{2}\right)}{2 \lambda^{2} f_{x} f_{y} f_{x y}},
\end{gathered}
$$

where $s$ is the scale factor associated with the Fourier blocks and is given by $s=\sqrt{\lambda f}$, with $f$ being the focal length of the spherical lens used to realize the Fourier transforms. Thus by control of the parameters of the lenses, it is possible to simulate sections of anamorphic free space with desired parameters.

If an input function $\bar{f}(\bar{x}, \bar{y})$ is given with its arguments in meters, we can then introduce the input

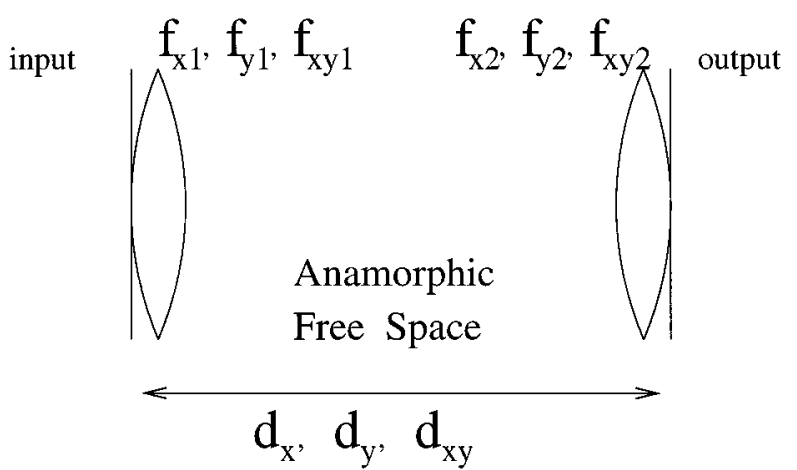

Fig. 3. Optical setup for realizing the nonseparable fractional Fourier transform.

scale factors $s_{x_{\mathrm{in}}}$ and $s_{y_{\text {in }}}$ and the output scale factors $s_{x_{\text {out }}}$ and $s_{y_{\text {out }}}$ to obtain the function $f(x, y)$ with dimensionless arguments, according to $f(x, y)=\bar{f}\left(s_{x_{\mathrm{in}}}, s_{y_{\mathrm{in}}}\right)$. The output is given by Eq. (6) in a form that again takes dimensionless arguments. It can easily be converted into a form whose arguments are in meters by substitution of $\left(\bar{x} / s_{x_{\text {out }}}, \bar{y} / s_{y_{\text {out }}}\right)$ for $(x, y)$.

With this understanding, the system shown in Fig. 3 will realize the nonseparable transform with the specified parameters $\phi_{1}, \phi_{2}, \theta_{1}, \theta_{2}, s_{x_{\text {in }}}, s_{x_{\text {out }}}$, and $s_{y_{\text {out }}}$ if we choose the design parameters $d_{x}, d_{y}, d_{x y}, f_{x 1}, f_{x 2}$, $f_{x y 1}, f_{x 2}, f_{y 2}$, and $f_{x y 2}$ according to the following:

$$
\begin{aligned}
d_{x} & =\frac{s_{x_{\text {in }}} s_{x_{\text {out }}} \cos \left(\theta_{1}-\theta_{2}\right)}{2 \lambda \cos \theta_{2} \csc \phi_{1}}, \\
d_{y} & =\frac{s_{y_{\text {in }}} s_{y_{\text {out }}} \cos \left(\theta_{1}-\theta_{2}\right)}{2 \lambda \cos \theta_{1} \csc \phi_{2}}, \\
d_{x y} & =\frac{s_{x_{\text {in }}} s_{y_{\text {out }}} \cos \left(\theta_{1}-\theta_{2}\right)}{2 \lambda \sin \theta_{2} \csc \phi_{2}}=\frac{-s_{y_{\text {in }}} s_{x_{\text {out }}} \cos \left(\theta_{1}-\theta_{2}\right)}{2 \lambda \sin \theta_{1} \csc \phi_{1}}, \\
\frac{1}{\lambda f_{x 1}} & =\frac{2 \cos \theta_{2} \csc \phi_{1}}{s_{x_{\text {in }}} s_{x_{\text {out }}} \cos \left(\theta_{1}-\theta_{2}\right)}-\frac{\cos ^{2} \theta_{2} \cos \phi_{1}+\sin ^{2} \theta_{2} \cot \phi_{2}}{s_{x_{\text {in }}}^{2} \cos ^{2}\left(\theta_{1}-\theta_{2}\right)}, \\
\frac{1}{\lambda f_{y 1}} & =\frac{2 \cos \theta_{1} \csc \phi_{2}}{s_{y_{\text {in }}} s_{y_{\text {out }}} \cos \left(\theta_{1}-\theta_{2}\right)}-\frac{\cos ^{2} \theta_{1} \cos \phi_{2}+\sin ^{2} \theta_{1} \cot \phi_{1}}{s_{y_{\text {in }}}^{2} \cos ^{2}\left(\theta_{1}-\theta_{2}\right)}, \\
\frac{1}{\lambda f_{x y 1}} & =\frac{2 \sin \theta_{2} \csc \phi_{2}}{s_{x_{\text {in }}} s_{y_{\text {out }}} \cos \left(\theta_{1}-\theta_{2}\right)}+\frac{2 \sin \theta_{1} \cos \theta_{2} \cot \phi_{1}-2 \cos \theta_{1} \sin \theta_{2} \cot \phi_{2}}{s_{x_{\text {in }}} s_{y_{\text {in }}} \cos ^{2}\left(\theta_{1}-\theta_{2}\right)}, \\
\frac{1}{\lambda f_{x 2}} & =\frac{2 \cos \theta_{2} \csc \phi_{1}}{s_{x_{\text {in }}} s_{x_{\text {out }}} \cos \left(\theta_{1}-\theta_{2}\right)}-\frac{\cot \phi_{1}}{s_{x_{\text {out }}}^{2}}, \\
\frac{1}{\lambda f_{y 2}} & =\frac{2 \cos \theta_{1} \csc \phi_{2}}{s_{y_{\text {in }}} s_{y_{\text {out }}} \cos \left(\theta_{1}-\theta_{2}\right)}-\frac{\cot \phi_{2}}{s_{y_{\text {out }}}^{2}}, \\
\frac{1}{\lambda f_{x y 2}} & =\frac{2 \sin \theta_{2} \csc \phi_{2}}{s_{x_{\text {in }}} s_{y_{\text {out }}} \cos \left(\theta_{1}-\theta_{2}\right)} .
\end{aligned}
$$






(a)

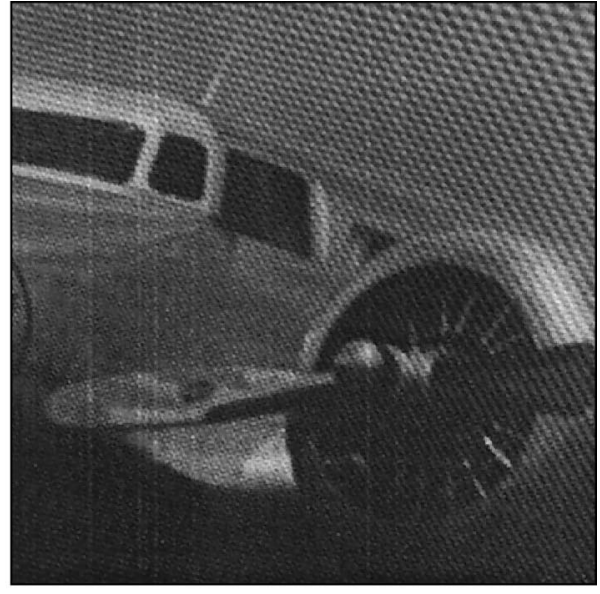

(b)

Fig. 4. (a) Original image. (b) Noisy image with a value of $\mathrm{SNR}=1$.

Unfortunately this system does not allow the specification of all four of the scale parameters $s_{x_{\mathrm{in}}}, s_{y_{\mathrm{in}}}$, $s_{x_{\text {out }}}$, and $s_{y_{\text {out }}}$ independently. If we specify $s_{x_{\text {in }}}$ and $s_{y_{\text {in }}}$, then $s_{x_{\text {out }}}$ and $s_{y_{\text {out }}}$ must be chosen in a way to satisfy the following:

$$
\frac{s_{x_{\text {out }}}}{s_{y_{\text {out }}}}=\frac{-s_{x_{\text {in }}} \sin \theta_{1} \csc \phi_{1}}{s_{y_{\text {in }}} \sin \theta_{2} \csc \phi_{2}} .
$$

If we specify $s_{x_{\text {out }}}$ and $s_{y_{\text {out }}}$ instead, then $s_{x_{\text {in }}}$ and $s_{y_{\text {in }}}$ must be chosen in a way to satisfy the following:

$$
\frac{s_{x_{\text {in }}}}{s_{y_{\text {in }}}}=\frac{-s_{x_{\text {out }}} \sin \theta_{2} \csc \phi_{2}}{s_{y_{\text {out }}} \sin \theta_{1} \csc \phi_{1}} .
$$

\section{Application to Signal Restoration}

Fractional Fourier domain filtering, ${ }^{2,32-38}$ has been applied successfully to the restoration of both 1-D and signals and 2-D images. Allowing different transform orders along the $x$ and the $y$ directions gives greater flexibility and results in smaller mean-square errors (MSE's) in the restoration of images. This flexibility is exploited in Ref. 39. However, in Ref. 39 the separable fractional Fourier transform is employed so that the fractional transform orders cannot be specified along arbitrary directions but are restricted along the orthogonal $x$ and $y$ axes. Here we show that allowing the transform orders to be specified along arbitrary directions results in greater flexibility and further reduction in estimation error.

Consider the following signal observation model:

$$
\mathbf{o}=\mathscr{H}(\mathbf{f})+\mathbf{n},
$$

where $\mathscr{H}(\cdots)$ is a linear system that degrades the desired signal $\mathbf{f}$ and $\mathbf{n}$ is an additive-noise term. Our problem is to recover $\mathbf{f}$ as closely as possible. The error criterion to be minimized is the MSE. It is assumed that the correlation functions of the input and the noise processes are known: $R_{f}\left(x, y ; x^{\prime}, y^{\prime}\right)=$ $E\left[f(x, y) f\left(x^{\prime}, y^{\prime}\right)\right], R_{n}\left(x, y ; x^{\prime}, y^{\prime}\right)=E\left[n(x, y) n\left(x^{\prime}, y^{\prime}\right)\right]$.
We first apply the fractional Fourier transform operator $\mathscr{F}_{\theta_{1}, \theta_{2}}^{a_{1}, a_{2}}$ to the observation o to transform it to the fractional Fourier domain characterized by the four parameters $a_{1}, a_{2}, \theta_{1}$, and $\theta_{2}$. Then we apply a multiplicative filter $\mathbf{g}$ in this domain. Finally, we inverse transform to the original space domain. Thus the estimate can be expressed in the form

$$
\hat{\mathbf{f}}=\left\{\mathscr{F}_{\theta_{1}, \theta_{2}}^{a_{1}, a_{2}}\right\}^{-1}\left[\mathbf{g} \cdot \mathscr{F}_{\theta_{1}, \theta_{2}}^{a_{1}, a_{2}}(\mathbf{o})\right],
$$

where $\left\{\mathscr{F}_{\theta_{1}, \theta_{2}}^{\left.a_{1}, a_{2}\right\}^{-1}}\right.$ is the inverse nonseparable fractional Fourier transform operator. The MSE is

$$
\sigma_{e}^{2}=E\left[|\mathbf{f}-\hat{\mathbf{f}}|^{2}\right] .
$$

Because the nonseparable fractional Fourier transform is unitary, this MSE is equal to the error in the transform domain. It can be shown by modification of the solution in Ref. 39 that the optimal filter function that minimizes the MSE is

$$
g_{\mathrm{opt}}(x, y)=\frac{R_{\tilde{f}, \tilde{o}}\left(x, y ; x^{\prime}, y^{\prime}\right)}{R_{\tilde{\tilde{o}, \tilde{o}}}\left(x, y ; x^{\prime}, y^{\prime}\right)} .
$$

In Eq. (39), $\tilde{f}$ and $\tilde{o}$ are the nonseparable fractional Fourier transforms of $f(x, y)$ and $o(x, y)$, respectively, with the parameters $a_{1}, a_{2}, \theta_{1}$, and $\theta_{2} . \quad R_{\tilde{f}, \tilde{o}}\left(x, y ; x^{\prime}\right.$, $\left.y^{\prime}\right)$ and $R_{\tilde{o}, \tilde{o}}\left(x, y ; x^{\prime}, y^{\prime}\right)$ are the correlation functions in the transform domain. They are defined as

$$
\begin{aligned}
R_{\tilde{f}, \tilde{o}}\left(x, y ; x^{\prime}, y^{\prime}\right) & =E\left[\tilde{f}(x, y) \tilde{o}\left(x^{\prime}, y^{\prime}\right)\right], \\
R_{\tilde{o}, \tilde{o}}\left(x, y ; x^{\prime}, y^{\prime}\right) & =E\left[\tilde{o}(x, y) \tilde{o}\left(x^{\prime}, y^{\prime}\right)\right] .
\end{aligned}
$$

These correlation functions can easily be calculated from the correlation functions in the space domain. The optimal choice of $a_{1}, a_{2}, \theta_{1}$, and $\theta_{2}$ are those that result in the minimum MSE.

We now consider an example that benefits from the use of the nonseparable transform. We choose a de- 


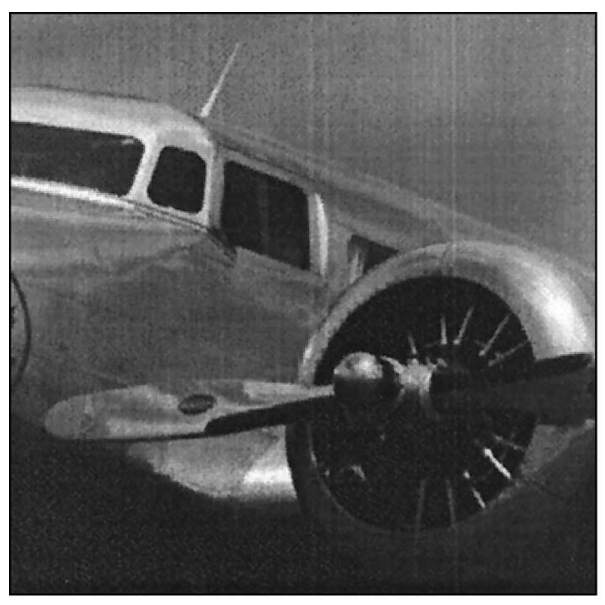

(a)

Fig. 5. Original image.

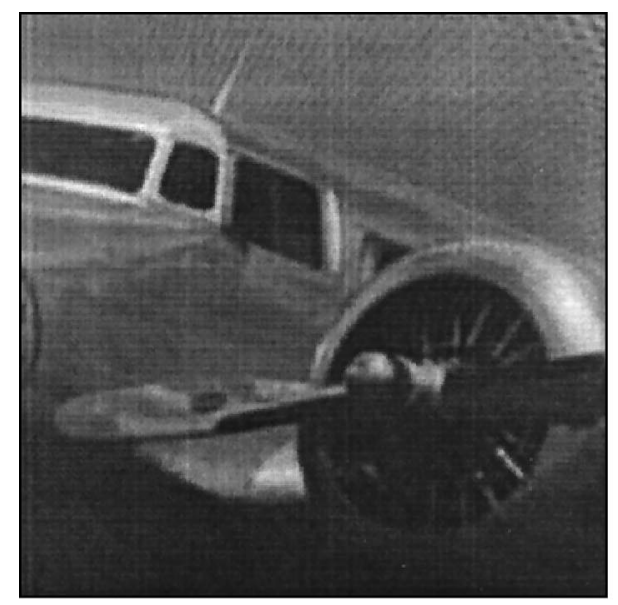

(a)

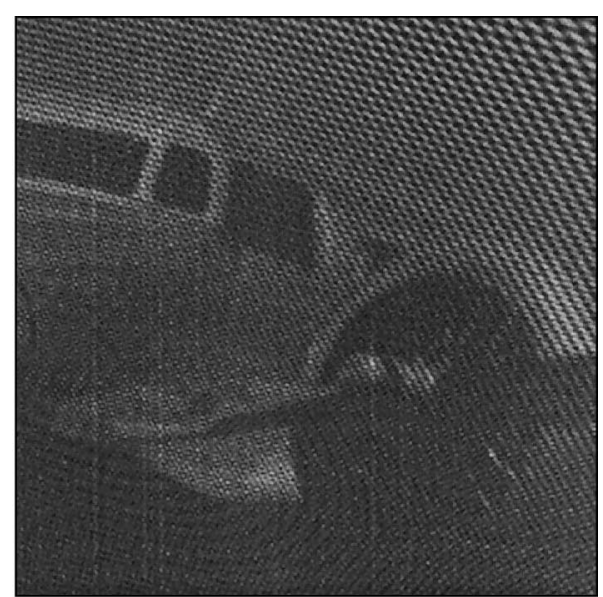

(b)

(b) Noisy image with a value of SNR $=0.1$.

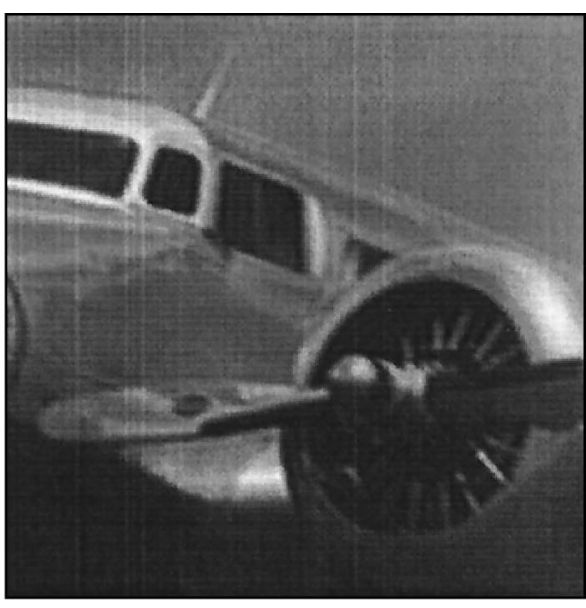

(b)

Fig. 6. Images with a value of SNR =1: (a) Image filtered by the separable transform. (b) Image filtered by the nonseparable transform.

terministic additive distortion with well-defined time-frequency characteristics:

$$
\begin{aligned}
f(x, y)+C\left\{\exp \left[1.6 i \pi\left(x^{\prime}-7.3\right)^{2}\right]\right. \\
\left.+\exp \left[1.4 i \pi\left(y^{\prime}+7.3\right)^{2}\right]\right\},
\end{aligned}
$$

where $f(x, y)$ is the desired image and $C$ is a constant that allows us to adjust the signal-to-noise ratio (SNR) to different values. The original and the distorted images can be seen in Figs. 4 and 5 .

The two chirps that constitute the distortion are not oriented along the $x$ and the $y$ directions but along arbitrary $x^{\prime}$ and $y^{\prime}$ directions. We consider two cases: $\quad$ SNR $=1$ and SNR $=0.1$. The SNR is defined as

$$
\mathrm{SNR}=\frac{\iint|f(x, y)|^{2} \mathrm{~d} x \mathrm{~d} y}{\iint|n(x, y)|^{2} \mathrm{~d} x \mathrm{~d} y}
$$

We compare the use of our nonseparable transform with the separable transform. The method in Ref. 39 makes use of the separable definition and minimizes the MSE by optimization over all possible combinations of $a_{1}$ and $a_{2}$. With the same approach the optimum transform orders are found to be $a_{1}=0.35$ and $a_{2}=-0.4$. (Remember that the separable transform is a special case of the nonseparable transform with $\theta_{1}=\theta_{2}=0$.) The restored images for $\mathrm{SNR}=1$ and $\mathrm{SNR}=0.1$ can be seen in Figs. 6(a) and 7(a), respectively.

When we use the filtering method based on the nonseparable transform, we optimize over $\theta_{1}$ and $\theta_{2}$ in addition to $a_{1}$ and $a_{2}$. The optimum parameters are found to be $\left(a_{1}=0.35, \theta_{1}=15^{\circ}\right)$ and $\left(a_{2}=-0.4\right.$, $\left.\theta_{2}=30^{\circ}\right)$. Figures $6(\mathrm{~b})$ and $7(\mathrm{~b})$ show the restored images for $\mathrm{SNR}=1$ and $\mathrm{SNR}=0.1$, respectively. (Because of computational constraints, we restrict our search to a local minimum only. It might be possible to obtain even better results by use of more sophisticated optimization methods.) 


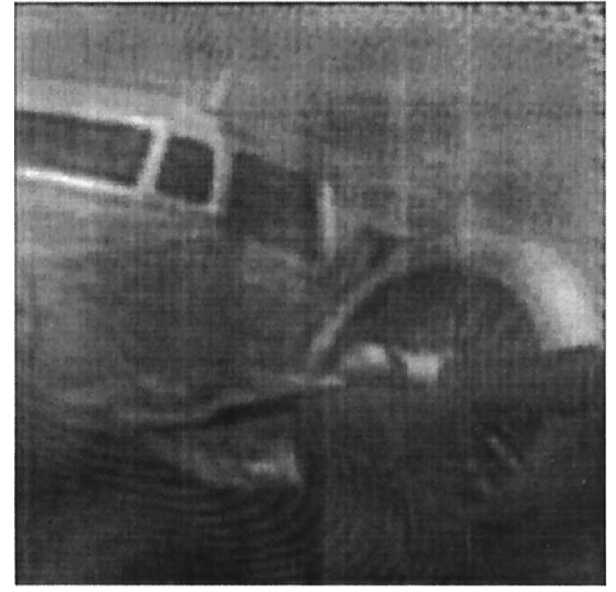

(a)

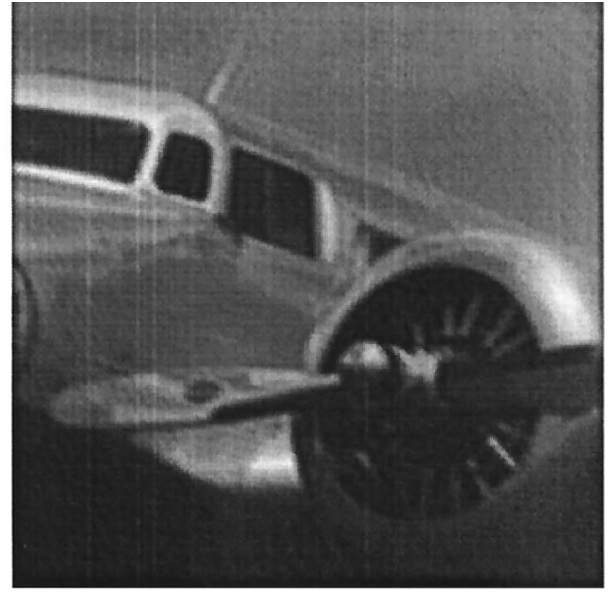

(b)

Fig. 7. Images with a value of $\mathrm{SNR}=0.1$ : (a) Image filtered by the separable transform. (b) Image filtered by the nonseparable transform.

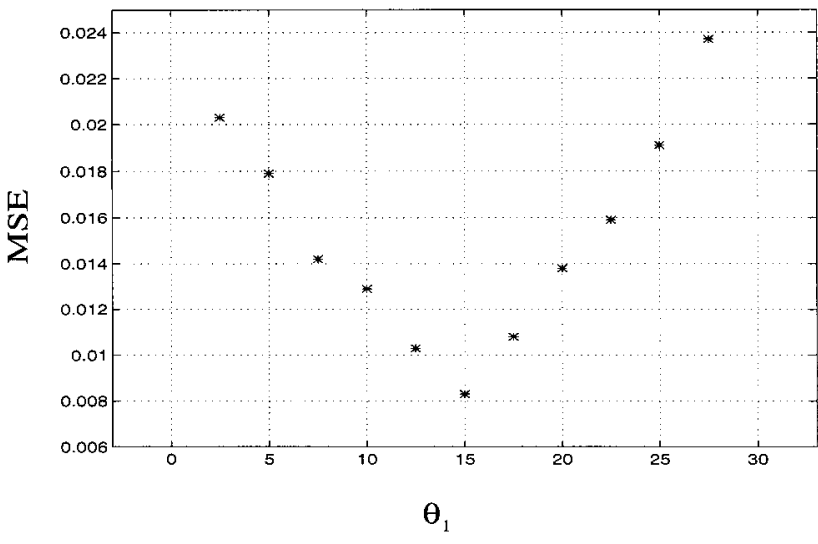

Fig. 8. Normalized MSE as a function of $\theta_{1}$ for a value of $\mathrm{SNR}=1$.

The improvement when SNR $=0.1$ is immediately visible when Figs. 7(a) and 7(b) are compared. In this case the nonseparable definition gives a MSE of 0.020 , whereas the separable definition results in a MSE of 0.101. Thus the MSE is reduced by a factor of 5 . When $\mathrm{SNR}=1$, the visible improvement is less evident, but nevertheless the MSE has decreased from 0.029 to 0.0084 , and the MSE is reduced by a factor of 3. (The MSE values given here are all normalized by the energy of the original image.)

Figures 8 and 9 illustrate the nature of the minimum point as they show how the MSE changes as either $\theta_{1}$ or $\theta_{2}$ is perturbed from its optimum value. We expect 2-D fractional Fourier domain filtering to find many applications in optical systems. This is because the types of distortion for which fractional Fourier domain filtering achieves the greatest benefits arise naturally in optical systems. For example, line defects on optical components produce a chirplike distortion. Because the angle between the defects will not necessarily be $90^{\circ}$, use of the nonseparable transform will result in greater improvements compared with use of the separable fractional Fourier transform and the ordinary Fourier transform.

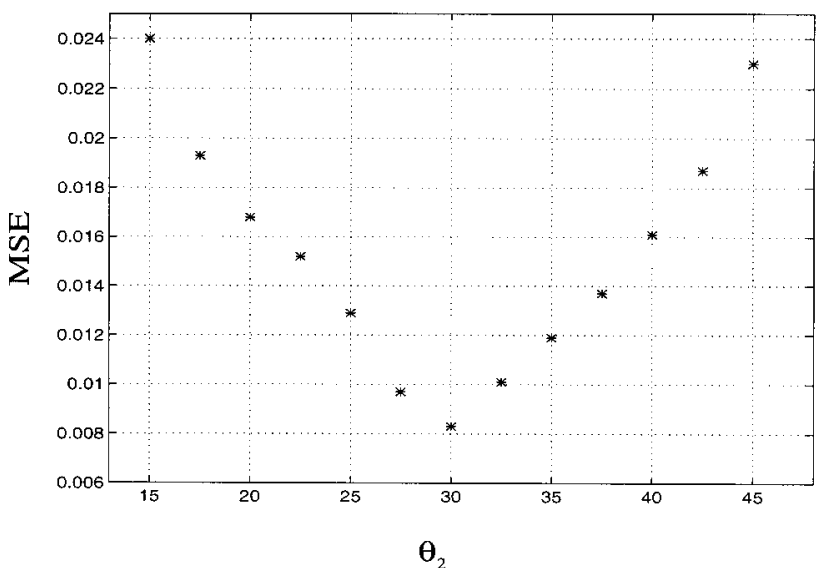

Fig. 9. Normalized MSE as a function of $\theta_{2}$ for a value of $\mathrm{SNR}=1$.

Such filtering schemes could also find application in optical systems to remove twin images in holography. ${ }^{40}$

\section{Conclusion}

The fractional Fourier transform has been generalized to two dimensions by application of the 1-D definition in the $x$ and the $y$ directions separately. Because the transform defined in this manner is separable, its properties are similar to that of the 1-D transform. The separable definition fails to satisfy certain properties that the common Fourier transform satisfies. When a linear distortion is applied to the function, its 2-D fractional Fourier transform cannot be represented as a linearly distorted version of the fractional Fourier transform of the original function. In this paper we have defined the nonseparable fractional Fourier transform for which it is possible to specify the transform orders along arbitrary directions without being constrained to the orthogonal $x$ and $y$ axes. We have also shown that an even more general definition is redundant because it can be expressed as a scaled version of the definition 
proposed in this paper. The digital and optical implementations of the nonseparable definition have been given, and its properties have been presented. Finally, we have considered an application that justifies our new definition.

\section{References}

1. A. C. McBride and F. H. Kerr, "On Namias's fractional Fourier transform," IMA J. Appl. Math. 39, 159-175 (1987).

2. H. M. Ozaktas, B. Barshan, D. Mendlovic, and L. Onural, "Convolution, filtering, and multiplexing in fractional Fourier domains and their relation to chirp and wavelet transforms," J. Opt. Soc. Am. A 11, 547-559 (1994).

3. H. M. Ozaktas and D. Mendlovic, "Fourier transforms of fractional order and their optical interpretation," Opt. Commun. 101, 163-169 (1993).

4. A. W. Lohmann, "Image rotation, Wigner rotation, and the fractional order Fourier transform," J. Opt. Soc. Am. A 10, 2181-2186 (1993).

5. H. M. Ozaktas and D. Mendlovic, "Fractional Fourier transforms and their optical implementation: II," J. Opt. Soc. Am. A 10, 2522-2531 (1993).

6. D. Mendlovic and H. M. Ozaktas, "Fractional Fourier transforms and their optical implementation: I," J. Opt. Soc. Am. A 10, 1875-1881 (1993).

7. A. W. Lohmann and B. H. Soffer, "Relationships between the Radon-Wigner and fractional Fourier transforms," J. Opt. Soc. Am. A 11, 1798-1801 (1994).

8. L. B. Almeida, "The fractional Fourier transform and timefrequency representations," IEEE Trans. Signal Process. 42, 3084-3091 (1994).

9. H. M. Ozaktas and D. Mendlovic, "Fractional Fourier optics," J. Opt. Soc. Am. A 12, 743-751 (1995).

10. P. Pellat-Finet and G. Bonnet, "Fractional order Fourier transform and Fourier optics," Opt. Commun. 111, 141-154 (1994).

11. L. M. Bernardo and O. D. D. Soares, "Fractional Fourier transforms and optical systems," Opt. Commun. 110, 517-522 (1994).

12. M. F. Erden, H. M. Ozaktas, A. Sahin, and D. Mendlovic, "Design of dynamically adjustable fractional Fourier transformer," Opt. Commun. 136, 52-60 (1997).

13. D. Mendlovic, Y. Bitran, C. Ferreira, J. Garcia, and H. M. Ozaktas, "Anamorphic fractional Fourier transformingoptical implementation and applications," Appl. Opt. 34, 74517456 (1995).

14. A. Sahin, H. M. Ozaktas, and D. Mendlovic, "Optical implementation of the two-dimensional fractional Fourier transform with different orders in the two dimensions," Opt. Commun. 120, 134-138 (1995).

15. P. Pellat-Finet, "Fresnel diffraction and the fractional-order Fourier transform," Opt. Lett. 19, 1388-1390 (1994).

16. L. M. Bernardo and O. D. D. Soares, "Fractional Fourier transforms and imaging," J. Opt. Soc. Am. A 11, 2622-2626 (1994).

17. T. Alieva, V. Lopez, F. Agullo-Lopez, and L. B. Almedia, "The fractional Fourier transform in optical propagation problems," J. Mod. Opt. 41, 1037-1044 (1994).

18. D. Mendlovic, Z. Zalevsky, N. Konforti, R. G. Dorsch, and A. W. Lohmann, "Incoherent fractional Fourier transform and its optical implementation," Appl. Opt. 34, 7615-7620 (1995).

19. R. G. Dorsch, "Fractional Fourier transformer of variable order based on a modular lens system," Appl. Opt. 34, 6016-6020 (1995).

20. A. W. Lohmann, "A fake zoom lens for fractional Fourier experiments," Opt. Commun. 115, 437-443 (1995).

21. C. C. Shih, "Optical interpretation of a complex-order Fourier transform," Opt. Lett. 20, 1178-1180 (1995).

22. R. G. Dorsh, A. W. Lohmann, Y. Bitran, D. Mendlovic, and H. M. Ozaktas, "Chirp filtering in the fractional Fourier domain,” Appl. Opt. 33, 7599-7602 (1994).

23. Y. B. Karasik, "Expression of the kernel of a fractional Fourier transform in elementary functions," Opt. Lett. 19, 769-770 (1994).

24. A. Sahin, "Two-dimensional fractional Fourier transform and its optical implementation," M.S. thesis (Bilkent University, Ankara, Turkey, 1996).

25. A. Sahin, H. Ozaktas, and D. Mendlovic, "Optical implementations of two-dimensional fractional Fourier transforms and linear canonical transforms with arbitrary parameters," Appl. Opt. 37, 2130-2141 (1998).

26. R. N. Bracewell, K. Y. Champ, A. K. Jha, and Y. H. Wang, "Affine theorem for two dimensional Fourier transform," Electron. Lett. 29, 304-309 (1993).

27. M. J. Bastiaans, "Wigner distribution function and its application to first-order optics," J. Opt. Soc. Am. 69, 1710-1716 (1979).

28. G. B. Folland, Harmonic Analysis in Phase Space (Princeton U. Press, Princeton, N.J., 1989.)

29. H. M. Ozaktas, O. Arikan, M. A. Kutay, and G. Bozdagi, "Digital computation of the fractional Fourier transform," IEEE Trans. Signal Process. 44, 2141-2150 (1996).

30. J. S. Lim, Two-Dimensional Signal and Image Processing (Prentice-Hall, Englewood Cliffs, N.J., 1990.)

31. Tanju Erdem. Bilkent University, Department of Electrical Engineering, 06533, Bilkent, Ankara, Turkey. (Personal communication.)

32. M. A. Kutay, H. M. Ozaktas, O. Arikan, and L. Onural, "Optimal filtering in Fractional Fourier domains," IEEE Trans. Signal Process. 45, 1129-1143 (1997).

33. M. F. Erden, H. M. Ozaktas, and D. Mendlovic, "Synthesis of mutual intensity distributions using the fractional Fourier transform," Opt. Commun. 125, 288-301 (1996).

34. A. W. Lohmann, Z. Zalevsky, and D. Mendlovic, "Synthesis of pattern recognition filters for fractional Fourier processing," Opt. Commun. 128, 199-204 (1996).

35. Z. Zalevsky and D. Mendlovic, "Fractional Wiener filter," Appl. Opt. 35, 3930-3936 (1996).

36. B. Barshan, M. A. Kutay, and H. M. Ozaktas, "Optimal filtering with linear canonical transformations," Opt. Commun. 135, 32-36 (1997).

37. H. M. Ozaktas, "Repeated fractional Fourier domain filtering is equivalent to repeated time and frequency domain filtering," Signal Process. 54, 81-84 (1996).

38. H. M. Ozaktas and D. Mendlovic, "Every Fourier optical system is equivalent to consecutive fractional Fourier domain filtering,” Appl. Opt. 35, 3167-3170 (1996).

39. M. A. Kutay and H. M. Ozaktas, "Optimal image restoration with the fractional Fourier transform," J. Opt. Soc. Am. A 15, 825-833 (1998).

40. L. Onural and P. D. Scott, "Digital decoding of in-line holograms," Opt. Eng. 26, 1124-1132 (1987). 